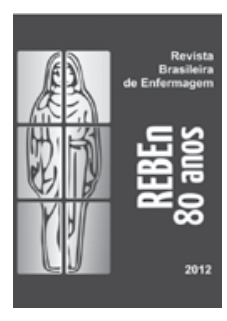

\title{
Classificação das áreas de conhecimento do CNPq e o campo da Enfermagem: possibilidades e limites
}

Classification of the CNPq areas of knowledge and the Nursing field: possibilities and limits

Clasificación de las áreas de conocimiento del CNPq y el campo de Enfermería: posibilidades y límites

\author{
Denize Cristina de Oliveira', Flávia Regina de Souza Ramos", \\ Alba Lucia Bottura Leite de Barros ${ }^{\text {III }}$ Maria Miriam Lima da Nóbrega ${ }^{\text {IV }}$ \\ ' Universidade do Estado do Rio de Janeiro, Faculdade de Enfermagem, Programa de Pós-Graduação em Enfermagem. \\ Rio de Janeiro-RJ, Brasil. Comitê de Assessoramento da Área de Enfermagem no CNPq. Brasília-DF, Brasil. \\ "Universidade Federal de Santa Catarina, Departamento de Enfermagem, Programa de Pós-Graduação em Enfermagem. \\ Florianópolis-SC, Brasil. Comitê de Assessoramento da Área de Enfermagem no CNPq. Brasília-DF, Brasil. \\ I'I Universidade Federal de São Paulo, Departamento de Enfermagem, Programa de Pós-Graduação em Enfermagem. \\ São Paulo-SP, Brasil. Comitê de Assessoramento da Área de Enfermagem no CNPq. Brasília-DF, Brasil. \\ Iv Universidade Federal da Paraíba, Departamento de Enfermagem de Saúde Pública e Psiquiatria, \\ Programa de Pós-Graduação em Enfermagem. João Pessoa-PB, Brasil. Comitê de Assessoramento \\ da Área de Enfermagem no CNPq. Brasília-DF, Brasil.
}

Submissão: 28-08-2013 Aprovação: 28-08-2013

\begin{abstract}
RESUMO
A classificação das áreas de conhecimento tem sido objeto de discussão por gestores e administradores de C\&T e pela comunidade científica na busca de instrumentos de apoio à sistematização de dados, principalmente para atividades de gestão e administração. A principal tabela de áreas em uso pelo CNPq está reconhecidamente desatualizada, necessitando de revisão ou reclassificação. O objetivo deste artigo é contribuir para o atual debate, com base em resultados prévios de participação em trabalho de revisão da tabela realizado na década de 1990 e de resultados de pesquisas na área da organização e representação do conhecimento no contexto da Ciência da Informação. Apresenta uma proposta de reformulação da Área de Conhecimento da Enfermagem e algumas reflexões sobre as possibilidades deste processo em curso.

Descritores: Conhecimento; Classificação; Política Nacional de Ciência, Tecnologia e Inovação; Enfermagem.
\end{abstract}

\begin{abstract}
Knowledge areas have been discussed by Science and Technology managers and administrators and by the scientific community searching for tools to support data systematization, mainly for management and administrative activities. The main table in use by $\mathrm{CNPq}$ is admittedly outdated and requires revision or reclassification. The aim of this article is to contribute to the current debate based on previous results from participation in a table review study performed in the 1990s and on research results in the area of knowledge organization and representation in the context of Information Science. It presents a proposal to reformulate the Nursing Knowledge Area and some reflections about the possibilities of this ongoing process.
\end{abstract}

Key words: Knowledge; Classification; National Science, Technology and Innovation Policy; Nursing. 


\section{RESUMEN}

La clasificación de las áreas de conocimiento viene siendo objeto de discusión por parte de los gestores y administradores de C\&T y por la comunidad científica en busca de instrumentos de apoyo a la sistematización de datos, principalmente para actividades de gerencia y administración. La principal tabla de áreas utilizada por el CNPq está reconocidamente desactualizada, necesitando de revisión o reclasificación. El objetivo de este artículo es contribuir para el actual debate sobre el asunto, con base en resultados previos de participación en trabajo de revisión de la referida tabla, realizado en la década de 1990, bien como de investigación en el área de la organización y representación del conocimiento en el contexto de la Ciencia de la Información. Se presenta una propuesta de reformulación del Área de Conocimiento de Enfermería y algunas reflexiones sobre las posibilidades de este proceso en curso.

Palabras clave: Conocimiento; Clasificación; Política Nacional de Ciencia, Tecnología e Innovación; Enfermería.

\section{AUTOR CORRESPONDENTEＡlba Lucia Bottura Leite de Barros E-mail: barros.alba@unifesp.br}

Trabalho originalmente apresentado no $17^{\circ}$ Seminário Nacional de Pesquisa em Enfermagem, Natal-RN, Associação Brasileira de Enfermagem Seção Rio Grande do Norte, 3 a 5 de junho de 2013

\section{INTRODUÇÃO}

Nos últimos trinta anos foram observadas importantes mudanças na ciência, decorrentes das novas doenças, dos novos sistemas de comunicação, como a internet e as novas tecnologias que resultaram na reconfiguração da própria vida social. O desenvolvimento do conhecimento na área de Enfermagem acompanhou a tendência mundial, refletindo os avanços necessários ao atendimento das novas demandas sociopolíticas e tecnológicas. Num mundo globalizado, no qual os conhecimentos são compartilhados em segundos, as ações e comportamentos de indivíduos e da sociedade devem, portanto ser compartilhados em tempo real. Isto se reflete na demanda relacionada às ações do cuidado, do ensino e consequente nas demandas de pesquisa na área de Enfermagem.

Decorrente dessas transformações, a atividade de pesquisa se tornou tão complexa e interdisciplinar que, muitas vezes, alocar alguns trabalhos em uma determinada área de conhecimento é tarefa difícil, que se faz de forma aproximativa apenas. A representação do universo do conhecimento em uma estrutura de classificação que permita organizar os dados da produção científica publicada e de outras atividades de Ciência, Tecnologia e Inovação (C\&TI) é o instrumento fundamental para as áreas de gestão e avaliação, assim como suporte representativo para a formulação, implementação, acompanhamento e avaliação de políticas públicas ${ }^{(1)}$.

A sistematização de dados na busca de indicadores de C\&TI sempre mereceu a atenção de organismos internacionais e, principalmente, daqueles países que apresentam índices representativos de produtividade em pesquisa e desenvolvimento tecnológico. O mapeamento criterioso e confiável do desenvolvimento de C\&TI para atender as diferentes perspectivas de demandas e interesses específicos de informação é considerado de importância inquestionável para gestores e administradores de C\&TI. É neste contexto que a tabela de classificação de "áreas do conhecimento" aparece como instrumento de apoio necessário e mesmo indispensável.

O Conselho Nacional de Desenvolvimento Cientifico e Tecnológico (CNPq) é uma agência do Ministério da Ciência,
Tecnologia e Inovação (MC\&TI) destinada ao fomento da pesquisa científica e tecnológica e à formação de recursos humanos para a pesquisa no país que adota determinada árvore de conhecimento como suporte para o desenvolvimento dos seus trabalhos.

Desde 1981 o CNPq vem atuando a partir do reconhecimento de nove grandes áreas do conhecimento. Mas é reconhecido, pelo próprio presidente do $\mathrm{CNPq}$, que é muito difícil mudar a árvore de áreas do conhecimento, pois sempre se faz reuniões, mas não se chega a conclusão alguma ${ }^{(2)}$. Entretanto, o desafio deve ser encarado, porque a divisão de áreas do $\mathrm{CNPq}$ reflete diretamente no modo como as pesquisas são avaliadas e no maior ou menor acesso a recursos.

As áreas do conhecimento podem ser definidas como "conjunto de conhecimentos inter-relacionados, coletivamente construído, reunido segundo a natureza do objeto de investigação com finalidades de ensino, pesquisa e aplicações práticas" $^{\prime(3)}$. A Tabela das Áreas do Conhecimento tem uma aplicação diversificada sendo adotada por órgãos atuantes em ciência, tecnologia, cultura, arte e inovação "é um instrumento para organizar informações visando implementar, administrar e avaliar seus programas e atividades [...] orienta os usuários dessas agências a situarem suas atividades no quadro geral da produção e aplicação do conhecimento" ${ }^{(4)}$.

Por sua vez, a complexidade de abordagem do universo de C\&TI e a diversidade de atividades desse sistema indicam a dificuldade de se construir, atualizar e compatibilizar instrumentos de classificação por áreas do conhecimento que satisfaçam os diferentes interesses institucionais de agregação de dados deste universo. Ressalta-se que a unidade do conhecimento é um tópico controvertido, principalmente em relação à divisão do conhecimento em disciplinas ${ }^{(5)}$.

No contexto da educação, Morin ${ }^{(6)}$ trata dos conceitos de disciplinaridade, interdisciplinaridade, polidisciplinaridade, transdisciplinaridade, multidisciplinaridade; e de expressões como mentalidade hiperdisciplinar, invasões interdisciplinares, migrações interdisciplinares, disciplinas híbridas, disciplinas agregadas, fronteiras disciplinares. A simples enumeração desses termos, de difícil definição e apreensão por serem de 
natureza polissêmica e imprecisa, mostra a complexidade em lidar com o universo do conhecimento, não só no contexto da educação, como também em questões de organização e representação do conhecimento em sistemas de recuperação de informação, onde as estruturas de classificação desempenham papel de fundamental importância ${ }^{(1)}$.

No Brasil, as "Áreas do Conhecimento" têm sido tema de discussão envolvendo gestores e administradores, as agências de fomento e avaliação, as sociedades científicas, os institutos de pesquisa e a própria comunidade científica.

Ao longo dos últimos trinta anos algumas propostas de mudanças foram elaboradas. A última, de 2005, propôs 21 novas grandes áreas, acrescentando sobremaneira a quantidade atual, o que representou uma dificuldade para a sua aprovação, conforme afirmou o Presidente do CNPq, Glaucius Oliva ${ }^{(7)}$, em reunião realizada em 2013: "Queremos reduzir a quantidade de áreas, e não aumentar".

A construção de uma tabela de áreas do conhecimento envolve aspectos básicos de organização, portanto, de classificação. Langridge ${ }^{(5)}$ aponta quatro elementos fundamentais relativos aos princípios de classificação, que podem contribuir para a presente discussão:

a) A classificação transforma impressões sensoriais isoladas e incoerentes em objetos reconhecíveis e padrões recorríveis

Numa analogia ao objetivo central de organização das áreas do conhecimento, pode-se reeditar essa premissa a partir da assertiva de que a classificação transforma dados isolados e incoerentes em informação reconhecível no âmbito de C\&TI e recorrível acerca do contexto científico do país.

\section{b) Toda classificação está relacionada a um propósito}

No contexto das áreas do conhecimento, o propósito central de organização é a sistematização de dados em C\&TI na busca de indicadores, como apoio a atividades de planejamento, gestão e avaliação.

c) Os mesmos objetos podem ser classificados de maneiras diferentes dependendo do propósito a que se destinam

Portanto, podem-se construir tantas tabelas de áreas do conhecimento quanto forem os diferentes propósitos de sua construção. A expectativa está em torno das possíveis perguntas a serem contempladas em função de determinada ordenação de dados, atendendo a demandas específicas que, necessariamente, podem ser as mais diversas.

\section{d) Não há substituto à classificação}

Independentemente do fato de se admitir que toda classificação é arbitrária e que nenhuma categorização é tão perfeita e completa que satisfaça de forma plena às inúmeras expectativas de retorno, admite-se que não há substituto à classificação. Portanto, é necessário um investimento sólido na construção, revisão ou reclassificação de uma tabela de "áreas do conhecimento" que represente com propriedade o atual desenvolvimento em C\&TI do país.

Consideradas na atualidade como Árvores do Conhecimento, a classificação das áreas desempenha um conjunto de funções, destacadamente como: instrumento fundamental para as áreas de gestão e avaliação; suporte representativo para a formulação, implementação, acompanhamento e avaliação de políticas públicas na área de C\&TI. Além disso, a divisão de áreas do CNPq reflete diretamente no modo como as pesquisas recebem recursos e são avaliadas; a complexidade de abordagem e diversidade de atividades do sistema de C\&TI; a dificuldade de se construir, atualizar e compatibilizar instrumentos de classificação por áreas do conhecimento, que satisfaçam os diferentes interesses institucionais de agregação de dados deste universo. Por fim, há que se reconhecer que existem pesquisas completamente distintas sendo avaliadas na mesma área.

Essas considerações levam a refletir e analisar as áreas do conhecimento como problema de interesse específico de organização e representação do conhecimento que envolve não apenas a Ciência da Informação, mas especialmente cada campo do conhecimento, uma vez que não se trata tão somente de um esforço de sistematização de dados, mas de melhor deixar transparecer o que cada área do conhecimento faz hoje e, especialmente, o que projeta para o seu futuro, permitindo induções de campos pouco explorados do conhecimento(1).

Este texto compreende três partes principais relacionadas à temática central da Classificação das Áreas do Conhecimento e, particularmente, da Enfermagem. A primeira trata de considerações sobre a tradicional tabela de áreas do conhecimento em uso pelo CNPq e pela CAPES, especialmente em torno dos resultados do trabalho de revisão realizado na década de 1990. Na segunda parte apresenta reflexão das autoras a partir da síntese de uma contribuição produzida por grupo de experts, composto por pesquisadores do CNPq e coordenadores de pós-graduação da área de Enfermagem, reunidos nas dependências do CNPq em 2013. A terceira parte sintetiza uma reflexão crítica sobre os desafios colocados a partir da proposição elaborada.

\section{ATUAL ÁRVORE DO CONHECIMENTO DO CNPQ}

A classificação das Áreas do Conhecimento em uso pelo CNPq e pela CAPES, é derivada especialmente dos resultados do trabalho de revisão realizado na década de 1990 e apresenta uma hierarquização em quatro níveis, que vão do mais geral aos mais específicos, abrangendo nove grandes áreas, 76 áreas e 340 subáreas do conhecimento ${ }^{(4)}$.

Define-se por "Grande área" a aglomeração de diversas áreas do conhecimento em virtude da afinidade de seus objetos, métodos cognitivos e recursos instrumentais refletindo contextos sociopolíticos específicos. Por "Sub-área" entende-se a segmentação da área do conhecimento estabelecida em função do objeto de estudo e de procedimentos metodológicos reconhecidos e amplamente utilizados. A "Especialidade" reflete a caracterização temática da atividade de pesquisa e ensino. Uma mesma especialidade pode ser enquadrada em diferentes grandes áreas, áreas e sub-áreas ${ }^{(3)}$. 
A Enfermagem, enquanto campo de conhecimento reflete-se na árvore atual da seguinte forma ${ }^{(4)}$ :

\subsection{Ciências da Saúde \\ 4.04 Subárea - Enfermagem \\ - Enfermagem Médico-Cirúrgica \\ - Enfermagem Obstétrica \\ - Enfermagem Pediátrica \\ - Enfermagem Psiquiátrica \\ - Enfermagem de Doenças Contagiosas \\ - Enfermagem de Saúde Pública}

O progresso observado no conhecimento de Enfermagem, no que diz respeito ao uso de estratégias que favoreçam o desenvolvimento científico, respondem às recomendações feitas à área por órgãos de fomento à pesquisa, como o CNPq e a CAPES e também pelo desenvolvimento histórico do Comitê Assessor de Enfermagem no CNPq e pela Representação de Área na CAPES. ${ }^{(8-12)}$.

Os pesquisadores, ao longo dos anos, foram se organizando em resposta as demandas buscando indagar e responder as novas realidades. Este fato vem de encontro ao que afirma Fourez $^{(13)}$, quando propõe que uma disciplina científica nasce como uma nova maneira de considerar o mundo e essa nova maneira se estrutura em ressonância com as condições culturais, econômicas, sociais e tecnológicas de uma época.

Não se pode deixar de reconhecer a influência do modelo biomédico no desenvolvimento histórico da Enfermagem e de suas práticas assistenciais, gerenciais e de formação, substituindo um movimento iniciado com Florence Nightingale para delinear um domínio próprio da Enfermagem. A era de total dependência deste modelo negligenciou o paciente como ser humano e o ambiente como algo significativo no cuidado de pessoas doentes, resultando em conceitualização e desenvolvimento de ideias que emanaram e influenciaram as práticas da Enfermagem ${ }^{(14)}$.

Neste processo, a Enfermagem desenvolveu um amplo movimento de crítica às bases citadas, especialmente no que se refere à herança do modelo biomédico na saúde e ao paradigma da ciência positivista. Entendendo-se o primeiro como modos de conhecer e intervir em saúde com impacto nas práticas e na formação profissional; e o segundo como lógica hegemônica e definidora de status científico. As mudanças em curso na formação profissional em Enfermagem já incorporaram avanços no sentido de superar os tradicionais ramos do conhecimento historicamente importados da medicina, o que ainda não se fez traduzir nas áreas de conhecimento.

O que se observa na classificação das áreas de conhecimento é, ainda, a influência do paradigma da medicina científica, reforçado pelo comumente denominado "modelo flexneriano de ensino médico", que consolidou, orientou e demarcou a educação superior para além da área médica, consagrando postulados como da excessiva fragmentação disciplinar dos saberes sobre a doença, da visão biologizante do vivido, da medicalização crescente do social, da orientação para o mercado, da especialização e da incorporação tecnológica. Embora haja discordâncias quanto a poder atribuir toda esta influência ao Relatório Flexner, desconhecendo diferenças nas formas deturpadas como o mesmo foi aplicado e retoricamente apropriado, não há duvidas quanto a hegemonia do modelo de ciência médica, independente da maior ou menor complexidade que se dê à análise de suas origens ${ }^{(15)}$.

Não foi apenas sobre a formação profissional que se estenderam as influências deste modelo e a atual árvore composta pelas áreas e sub-áreas de conhecimento explicita a adesão ao modo clássico de nomear os campos de produção de conhecimento, segundo as especialidades médicas, demonstrando o quão lenta tem sido a superação desse modelo. Exemplo desse processo pode ser observado na denominação "Enfermagem médico-cirúrgica", apontando para um conjunto de patologias (clinicas e cirúrgicas), para serviços hospitalares, para uma especialidade médica. Enfim, o que cabe problematizar é o que estes termos dizem sobre nós e para nós nos dias atuais e não do nosso passado.

Os produtos das pesquisas de enfermagem, desenvolvidas na última década, associados aos argumentos tecidos apontam a necessidade de revisão da classificação atualmente adotada da área do conhecimento da Enfermagem. Uma vez que as mesmas não contemplam, não caracterizam e não evidenciam os objetos estudados na atualidade da Enfermagem, e da mesma forma não projetam os desafios colocados no futuro próximo para o desenvolvimento da área. Deve-se ressaltar que as alterações nas denominações das áreas de conhecimento trazem consigo elementos conceituais, e não apenas semânticos, que implicam mudanças na própria identidade do campo de pesquisa, especialmente considerando as mudanças nas políticas de saúde, no foco do cuidado de enfermagem enfatizando os grupos e os sujeitos do cuidado e nos problemas nacionais.

\section{PROPOSTA DE REFORMULAÇÃO DA ÁREA DE CONHE- CIMENTO DA ENFERMAGEM}

Comprometidas com o avanço da ciência e sensíveis aos apelos frequentes das pesquisadoras da área de Enfermagem, manifestados em reuniões científicas organizadas pela CAPES, CNPq, Associação Brasileira de Enfermagem (ABEn) e Sociedades de Especialistas, os membros do Comitê de Assessoramento da Área de Enfermagem do CNPq (CA-EF) organizaram uma seminário em Brasília, em maio de 2013, onde uma das pautas foi a discussão e análise das sub-áreas da Enfermagem, com vistas à elaboração de uma proposta de reorganização das mesmas, a ser apresentada a Diretoria de Ciências Agrárias, Biológicas e da Saúde (DABS/CNPq).

Participaram deste seminário oitenta especialistas, pesquisadores do CNPq e coordenadores de Programas de Pós-Graduação em Enfermagem, de diferentes regiões do país. A metodologia do trabalho se deu por meio de grupos de discussão de especialistas, visando à análise crítica das atuais sub-áreas e apresentação de propostas de modificações.

Foram apresentadas diversas sugestões de alterações e acréscimos nas subáreas de conhecimento da Enfermagem, 
contemplando aspectos relacionados a administração, gestão e gerenciamento; saúde coletiva e saúde populacional; saúde do adulto, do idoso, senescência e senilidade; saúde da criança e do adolescente; saúde da mulher; saúde da família; saúde mental e psiquiatria; enfermagem fundamental, incluindo história, ética, filosofia, comunicação e epistemologia; tecnologia e inovação na Enfermagem; trabalho e educação na Enfermagem. Tendo em vista a amplitude e a diversidade de orientações das propostas apresentadas, buscou-se identificar as confluências dos objetos de pesquisa e também aderência as nomenclaturas adotadas nas políticas públicas e no contexto acadêmico.

Depois de realizadas as discussões, as recomendações dos seis grupos de trabalho para a área de Enfermagem foram apresentadas e discutidas em uma plenária, resultando na síntese apresentada abaixo. A proposta consensuada propõe a especificação da Área de Enfermagem em sete sub-áreas, sendo três circunscritas por grupo populacional e sujeitos do cuidado e quatro outras definidas por campo de especialidade.

4.00 Ciências da Saúde

4.04 Subárea - Enfermagem

- Enfermagem em Saúde do Adulto e Idoso

- Enfermagem em Saúde da Mulher

- Enfermagem em Saúde da Criança e do Adolescente

- Enfermagem em Saúde Mental

- Enfermagem em Saúde Coletiva

- Enfermagem Fundamental

- Enfermagem na Gestão e Gerenciamento

Essas sub-áreas são as que apresentaram maior expressão na produção do conhecimento de Enfermagem em termos do seus objetos de investigação nos últimos $\operatorname{anos}^{(16)}$, além de refletir os conceitos aderentes a esse campo de conhecimentos. A proposição apresentada pautou-se em alguns princípios e eixos organizadores, que são os seguintes: a) principio da autonomização de campos de conhecimento (relativo ao empenho de cada área em tornar-se distinta e peculiar, produzindo pautas, regras e cultura científica própria, apesar da adesão a uma episteme); b) princípio da realidade (problemas e necessidades práticas e temporalmente localizadas que demandam conhecimentos); c) princípio epistemológico (conhecimento buscado e o que se considera resultado legitimo de pesquisa, estratégias para a busca e os meios reconhecidos como válidos). O último princípio adotado, c) de campos emergentes, não pode ser concebido fora da relação dos três primeiros, pois é da relação entre realidade, justificação do conhecimento e movimentos de comunidades cientificas que emergem novos campos, como produtos de sociedades e momentos históricos, como no momento atual. Novos campos de conhecimento emergem de lacunas deixadas por outros campos pré-existentes ou por alianças entre eles; em fronteiras estendidas ou invadidas; em terrenos disputados ou inexplorados.
Empreender este exercício crítico aplicado à Enfermagem permite reconhecer não uma, mas diversas tendências em interação. Para a proposição das subáreas apresentadas foram considerados, além dos quatro princípios, alguns eixos derivados das relações entre os princípios assinalados. O eixo que valoriza os "fazeres e linguagens", ou seja, as formas próprias da Enfermagem expressar-se socialmente como um modo de fazer e dizer, que representa sua compreensão sobre os objetos do seu saber/fazer, em discursos próprios e politicamente situados. Também se pode reconhecer o eixo das "teorias e metodologias", que potencializado pelo anterior, aposta na constituição de linguagens, normas e tecnologias (sistemas, modelos, classificações, conceitos métodos, procedimentos) capazes de tornar mais circuláveis e extensivos os produtos do conhecimento.

Quaisquer que sejam as escolhas, o confronto com o desafio de estabelecer interfaces e convergência de áreas temáticas se coloca, reconhecendo tanto as demandas por verticalidade, como por transversalidade; tanto por especialização e desenvolvimento da disciplina de Enfermagem, como por interdisciplinaridade. Ambas são demandas legítimas, pois não se pode escapar das margens e critérios que dão acesso ao espaço da ciência, que impõe regras nem sempre pertinentes às especificidades da área, ou seja, é inegável o esforço por consolidar-se como disciplina científica.

\section{ALGUMAS REFLEXÕES SOBRE AS POSSIBILIDADES PARA A RECLASSIFICAÇÃO DA ÁREA DE CONHECIMENTO DA ENFERMAGEM}

O processo de revisão e atualização permanente de áreas, linhas e prioridades de pesquisa é inerente à produção do conhecimento e, embora com nuances distintas e guardando margem de diversificação e independência de campos específicos, é orientado pela episteme de uma época e por tendências relativamente comuns aos diferentes campos de conhecimento.

Diversos elementos ou movimentos atuam simultaneamente na conformação destas tendências, como forças que tencionam as áreas de conhecimento e mobilizam respostas articuladas pelo conjunto dos atores inseridos na CT\&l, especialmente pesquisadores e consumidores de conhecimento.

Muitas foram às profissões que fizeram o caminho da explicação - intervenção - conquista de monopólios de conhecimento. Para isto, foi fundamental o estabelecimento de margens seguras e vigiadas de usufruto e manejo de um instrumental esotérico, disponível apenas aos credenciados. Conflitos, defesa de fronteiras, crescente especialização e legitimação de saberes e práticas exclusivas da comunidade de pensamento são comuns neste cenário que, a partir da epistemologia de Fleck, pode ser visto como território com fronteiras "contingentes e negociáveis". Neste terreno ocorrem traduções de saberes de um estilo ou comunidade para outro, não como simples importação, mas enriquecendo e atribuindo novas qualidades ao traduzido ${ }^{(17)}$.

Uma opção para pensar focos possíveis na demarcação de subáreas, tal como o "foco no cuidado", ele próprio abordado como ação definidora da profissão, como objeto epistemológico da Enfermagem, como essência da disciplina Enfermagem, enfim, em suas múltiplas dimensões, incluindo a 
ético-política. No entanto, essa não foi a opção adotada nesta proposta de classificação, uma vez considerados os limites institucionais colocados pelo CNPq para este trabalho.

Também poderiam ser pautados os seguintes focos: - nos grupos ou sujeitos do cuidado (mulheres, crianças, adultos, idosos, adolescentes, entre outros); - nos problemas nacionais transversais (desde grandes temas como doenças crônicas, saúde mental, álcool e drogas, doenças endêmicas ou sexualmente transmissíveis, entre outros); - nas competências profissionais (administração/gestão, educação em Enfermagem, entre outras derivações); - na produção tecnológica do cuidado (modelos, classificações, tecnologias de informação, inovação, entre outras).

Por outro lado, o intercâmbio com outras áreas do conhecimento, tão necessário ao próprio avanço do conhecimento científico da Enfermagem mostra-se como desafio e risco. Os modelos e teorias próprios da Enfermagem são necessários, tanto quanto as teorias e referenciais auxiliares e retraduzidos para a compreensão dos objetos da Enfermagem. Assim como, é preciso circunscrever uma linguagem própria que dê identidade ao campo de conhecimento. $\mathrm{O}$ desafio se coloca entre cerrar as fronteiras e enfrentar o risco de perda de identidade, esvanecendo-se em indeterminação dos campos teóricos e objetos de estudo; entre fluidez e rigidez; entre abertura e clausura.

Finalmene, cabe assinalar que este desafio não é exclusivo da Enfermagem, mas tipicamente do momento atual de desenvolvimento da ciência que procura responder a desafios típicos de uma realidade fluida. Compartilhando o projeto moderno de vida comprometida com a plenitude de sentidos - autonomia, saúde, dignidade, justiça - a Enfermagem assume o princípio de responsabilidade, quase como uma condição que recoloca continuamente o questionamento e a tarefa do esclarecimento kantiano, retomada por Foucault. Saber até onde se é capaz de saber, saber até onde se pode raciocinar sem perigo; enfim, saber que a liberdade e a potência crítica está menos no que enfrentamos, e mais na ideia que se faz do próprio conhecimento e dos seus limites ${ }^{(18)}$.

\section{REFERÊNCIAS}

1. Souza, R.F. Áreas do Conhecimento. Data Grama Zero Revista de Ciência da Informação. 2004; 5(2):53-70.

2. Oliva G. 60 anos do CNPq: avanços obtidos e perspectivas de ações futuras. In: $63^{\text {a }}$ Reunião Anual da SBPC. 2011 jul 10-15; Goiânia, Brasil.

3. Conselho Nacional de Desenvolvimento Científico e Tecnológico [homepage na internet]. Áreas de conhecimento. 2013 [acesso em 05 ago 2013]. Disponível em: http:// memoria.cnpq.br/areasconhecimento/index.htm

4. Conselho Nacional de Desenvolvimento Científico e Tecnológico [homepage na internet]. Dados abertos: áreas de conhecimento. 2013 [acesso em 05 ago 2013]. Disponível em: http://www.cnpq.br/web/guest/dados_abertos

5. Langridge DW. Classificação: abordagem para estudantes de biblioteconomia. Tradução de Rosali P. Fernandez. Rio de Janeiro: Interciência; 1977.

6. Morin E. A cabeça bem-feita: repensar a reforma, reformar o pensamento. Rio de Janeiro: Bertrand Brasil; 2001. p.128.

7. Conselho Nacional de Desenvolvimento Científico e Tecnológico[homepage na internet]. Seminário de avaliação debolsistas de produtividade. 2013 [acesso em 15 ago 2013]. Disponível em: http://www.cnpq.br/web/guest/noticiasviews/-/ journal_content/56_INSTANCE_a6MO/10157/955147

8. Mendes IAC. Pesquisa em enfermagem: impacto na prática. São Paulo: EDUSP; 1991.

9. Gutierrez MGR, Leite JL, Erdmann AL, Pagliuca LMF. Os múltiplos problemas pesquisados e a pesquisar na enfermagem. Rev Bras Enferm 2002;55(5):535-41.
10. Mendes IAC, Leite JL, Trevizan MA, Santos RM. Classificação dos pesquisadores/consultores da área de enfermagem no CNPq: contribuição para um banco de dados. Rev Bras Enferm 2003;56(5):488-93.

11. Barreira IA. A pesquisa em enfermagem no Brasil e sua posição em Agência Federal de Fomento. Rev Latino-Am Enferm1993;1(1):51-7.

12. Leite JL, Mendes IAC. Pesquisa em enfermagem e seu espaço no CNPq. Esc Anna Nery Rev Enferm 2000;4(3):389-94.

13. Fourez G. O método científico: a ciência como disciplina intelectual. In: Fourez G. A construção das ciências. Introdução à filosofia e a ética científica. São Paulo: Ed. Unesp; 1995.

14. Meleis Al. Theoretical Nursing: development e progress. 4th ed. LWW: [s.n]; 2007.

15. Almeida Filho N. Reconhecer Flexner: inquérito sobre produção de mitos na educação médica no Brasil contemporâneo. Cad Saúde Pública 2010;26(12):2234-49.

16. Oliveira DC. Os objetos de pesquisa predominantes nos projetos de pesquisa apresentados ao CNPq no período 2010-2012. Revista Enfermagem UERJ 2013.

17. Ramos FRS, Do OJ. Bioethics and professional identity - the healthcare worker's constrution of the experience of him/ herself. Interface - Comunic Saúde Educ 2009;13(28):1xxx.

18. Foucault M. O que são as luzes? In: Foucault Michel. Ditos e escritos: arqueologia das ciências e história dos sistemas de pensamento. Rio de Janeiro: Forense; 2000. p.335-351. 